\title{
Reduced-Complexity Bandwidth-Constrained Distributed Estimation for Wireless Sensor Networks
}

\author{
Trent Jacobs, Student Member, IEEE, Hlaing Minn, Member, IEEE, Naofal Al-Dhahir, Senior Member, IEEE \\ University of Texas at Dallas \\ (taj016000@utdallas.edu, hlaing.minn@utdallas.edu, aldhahir@utdallas.edu)
}

\begin{abstract}
While elegant in form, the maximum likelihood estimator (MLE) for heavily bandwidth-constrained distributed estimation in Gaussian noise is computationally expensive to implement. We consider an alternative estimator for this case which requires far less computational complexity, yet performs close to the MLE under the same operating conditions.
\end{abstract}

\section{INTRODUCTION}

As the size and power requirements of computational devices have decreased in the last fifteen years, research attention has been given to harnessing the distributed information which networks of small computationally-weak, battery-powered devices may collect [1]. Wireless sensors must generally operate on a small fixed-power source, such as a battery, and therefore demand computationally-efficient algorithms and low power transmission schemes for prolonged operation [2] [3]. Ribeiro and Giannakis have addressed both the issue of computationally-efficient detection at the sensors and the issue of low power transmission in [4] for the case of a deterministic signal in white Gaussian noise by limiting the sensors to only making binary decisions on the received data. When each sensor makes only one binary decision on the received data, the required transmission bandwidth (and therefore transmission power) is minimized for a sensor, as only one bit of information must be transmitted to the fusion center.

In this work we investigate the results in [4] under the condition in which all of the sensors in a network share a common threshold value for making binary decisions. While the estimator proposed in [4] for this case performs quite well, it is not computationally efficient, and as such places unnecessary computational burden on the fusion center. We consider an alternative estimator for this case which greatly reduces the computational complexity at the fusion center while providing comparable performance over the considered estimation range.

In Section II, we present a detailed description of and motivation for the work undertaken in this paper. In Section III, we derive our computationally-efficient estimator, and in Section IV we provide simulation results which illustrate the performance of our estimator. Finally, we offer concluding remarks in Section V.

\section{Problem Statement}

In [4], Ribeiro and Giannakis consider a bandwidthconstrained distributed estimation problem in which a network of sensors receive a deterministic signal corrupted by white Gaussian noise. Each sensor in the network quantizes the received signal-plus-noise on a series of threshold values, resulting in a sequence of binary observations. These binary observations (the bandwidth-constrained portion of the problem) are then transmitted to a fusion center which estimates the deterministic parameter by way of these binary sequences. The most stringent bandwidth constraint is the case of all sensors in the network using only one (identical) threshold value. The present work will consider this case. Maintaining consistency with the notation in [4], let $x(n)=\theta+w(n)$, where $w(n)$ is real-valued white Gaussian noise with variance $\sigma^{2}$. Each of the $N$ sensors in the network receives and quantizes $x(n)$ based on the threshold $\tau$, such that

$$
b(n)= \begin{cases}1, & x(n) \in[\tau,+\infty) \\ 0, & x(n) \in(\infty, \tau) .\end{cases}
$$

It is clear from this definition that each $b(n)$ is a Bernoulli trial with parameter

$$
\operatorname{Pr}\{b(n)=1\}=F(\tau-\theta) \triangleq p(\theta)
$$

where $F(x)$ is the complimentary Gaussian cumulative distribution function (CDF) defined by:

$$
F(x)=\frac{1}{\sqrt{2 \pi \sigma^{2}}} \int_{x}^{+\infty} \exp \left(\frac{-u^{2}}{2 \sigma^{2}}\right) d u .
$$

The quantized samples, $\{b(n)\}$, are transmitted to a central fusion center where the parameter $\theta$ is estimated. The received probability mass distribution function (pdf) at the fusion center is given by

$$
p(\mathbf{b} ; \theta)=\prod_{n=0}^{N-1} p(\theta)^{b(n)}(1-p(\theta))^{1-b(n)} .
$$

Additionally, the estimator given by

$$
\bar{x}=\frac{1}{N} \sum_{n=0}^{N-1} x(n)
$$

will be referred to as the clairvoyant estimator, which serves as a convenient benchmark for comparison of all bandwidthconstrained distributed estimators. Because the clairvoyant 
estimator operates on the noisy signal directly, instead of operating on the quantized samples of the noisy signal as the considered bandwidth-constrained estimators do, the clairvoyant estimator should generally outperform bandwidth-constrained estimators for $\theta$.

1) Cramer-Rao Lower Bound: The variance of any unbiased estimator of $\theta$ based on the observation vector $\mathbf{b}$ is lower bounded by the CRLB derived from the pdf $p(\mathbf{b} ; \theta)$. As such, it is useful to provide a derivation of the CRLB here for later use in performance evaluations of the estimators based on $\mathbf{b}$. Let $\mathbf{L}$ represent the natural $\log$ of (4). Then, we have:

$$
\begin{aligned}
& \frac{\partial \mathbf{L}}{\partial \theta}=\sum_{n=0}^{N-1}\left(\frac{b(n)}{p(\theta)} \frac{\partial p(\theta)}{\partial \theta}-\frac{1-b(n)}{1-p(\theta)} \frac{\partial p(\theta)}{\partial \theta}\right) \\
& \frac{\partial^{2} \mathbf{L}}{\partial \theta^{2}}=\sum_{n=0}^{N-1} \frac{b(n)}{p(\theta)}\left(\frac{-1}{p(\theta)}\left(\frac{\partial p(\theta)}{\partial \theta}\right)^{2}+\frac{\partial^{2} p(\theta)}{\partial \theta^{2}}\right) \\
& +\frac{1-b(n)}{1-p(\theta)}\left(\frac{-1}{1-p(\theta)}\left(\frac{\partial(1-p(\theta))}{\partial \theta}\right)^{2}+\frac{\partial^{2}(1-p(\theta))}{\partial \theta^{2}}\right) .
\end{aligned}
$$

For convenience, define $\dot{p}(\theta)=\frac{\partial p(\theta)}{\partial \theta}$. Then, we can obtain the CRLB as:

$$
\begin{aligned}
\mathrm{CRLB} & =\left\{-E\left[\frac{\partial^{2} \mathbf{L}}{\partial \theta^{2}}\right]\right\}^{-1} \\
& =\left\{\frac{N p(\theta)}{p(\theta)}\left(\frac{\dot{p}(\theta)^{2}}{p(\theta)}\right)+\frac{N(1-p(\theta))}{1-p(\theta)}\left(\frac{\dot{p}(\theta)^{2}}{1-p(\theta)}\right)\right\}^{-1} \\
& =\left\{N\left[\frac{\dot{p}(\theta)^{2}}{p(\theta)(1-p(\theta)}\right]\right\}^{-1} \\
& =\frac{p(\theta)(1-p(\theta)}{N \dot{p}(\theta)^{2}} \leq \operatorname{var}(\hat{\theta})
\end{aligned}
$$

2) Maximum Likelihood Estimator: From (4), the loglikelihood function is given by

$$
L(\theta)=\sum_{n=0}^{N-1} b(n) \ln (p(\theta))+(1-b(n)) \ln (1-p(\theta))
$$

which can be maximized, producing the MLE, as follows:

$$
\begin{aligned}
\frac{\partial \ln (p(\mathbf{b}, \theta))}{\partial \theta} & =\sum_{n=0}^{N-1}\left(\frac{b(n)}{p(\theta)} \frac{\partial p(\theta)}{\partial \theta}-\frac{1-b(n)}{1-p(\theta)} \frac{\partial p(\theta)}{\partial \theta}\right)=0 \\
0 & =\frac{\partial p(\hat{\theta})}{\partial \hat{\theta}} \sum_{n=0}^{N-1}\left(\frac{b(n)}{p(\hat{\theta})}-\frac{1-b(n)}{1-p(\hat{\theta})}\right) \\
0 & =\sum_{n=0}^{N-1}\left(\frac{b(n)(1-p(\hat{\theta}))+(1-b(n)) p(\hat{\theta})}{(1-p(\hat{\theta})) p(\hat{\theta})}\right) \\
0 & =\sum_{n=0}^{N-1}(b(n)-p(\hat{\theta})) \\
p(\hat{\theta}) & =\frac{1}{N} \sum_{n=0}^{N-1} b(n) \\
\hat{\theta}_{M L E} & =\tau-F^{-1}\left(\frac{1}{N} \sum_{n=0}^{N-1} b(n)\right)
\end{aligned}
$$

where we have used (2).

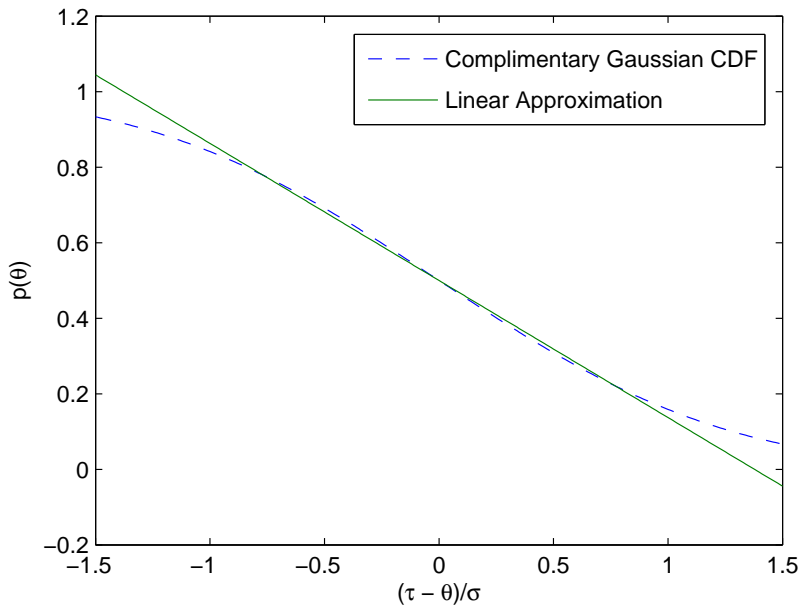

Fig. 1. The complimentary Gaussian CDF and the linear approximation

\section{A SiMPLER ESTIMATOR}

As shown in [4], the performance of the MLE is close to the CRLB when $\left|\frac{\tau-\theta}{\sigma}\right| \leq 1$. However, due to the reliance upon the inverse complimentary Gaussian CDF, the MLE is quite computationally expensive to implement at the fusion center. Either evaluation of a non-trivial integral or a table look-up with interpolation is required for evaluation of the inverse complimentary Gaussian CDF. If a table look-up is the implementation method chosen for the inverse complimentary Gaussian CDF required by the MLE, the "MLE" estimator becomes biased which results in a loss of guaranteed optimality. A table lookup requires a number of comparisons and interpolation calculations to produce a (biased and suboptimal) result. Thus, it is beneficial to consider a computationallyefficient approximation to the inverse complimentary Gaussian $\mathrm{CDF}$ for use at the fusion center.

\section{A. Linear Approximation}

The decision to use a linear approximation to the complimentary Gaussian CDF is motivated by both the observation that the function is nearly linear in the range $\left(-\frac{\tau-\theta}{2 \sigma}, \frac{\tau-\theta}{2 \sigma}\right)$, and the determination in [4] that the one bit per observation common threshold MLE is only suitable in applications for which there is high confidence that $\tau-\theta \leq \sigma$. By applying the least-squares linear regression technique to the complimentary Gaussian CDF in the range $\left|\frac{\tau-\theta}{\sigma}\right|$, we can find slope $A$ and intercept $B$ that best fit the curve in this region. A numerical evaluation gives $A=-0.36292$ and $B=0.5$. Fig. 1 illustrates the fit of this linear approximation to the original curve.

\section{B. The New Estimator}

The linear approximation to the inverse complimentary Gaussian CDF is of practical benefit if we consider an indirect estimate of $\theta$. That is, if we first obtain an estimate of the parameter, $p(\theta)$, of the binomial random variables which represent the quantized samples, $\{b(n)\}$, and then apply $p^{-1}(\theta)$ to obtain an estimate of $\theta$. This inverse function is of utility 
because $p(\theta)$ is a monotonic, isomorphic function of $\theta$ for both the inverse complimentary Gaussian CDF curve and our linear approximation to the curve. Thus, despite the lack of any guarantee of optimality in the derived estimate of $\theta$, we can be assured that each unique estimate of $p(\theta)$ will yield a corresponding unique estimate of $\theta$. Effectively, we consider the following expression

$$
p(\theta) \approx \frac{A}{\sigma}(\tau-\theta)+B, \quad|\tau-\theta| \leq \sigma
$$

for the inverse mapping of $p(\theta)$, instead of the inverse mapping of the complimentary Gaussian CDF. Simple algebraic manipulation then yields an estimator for $\theta$, with a compromise of optimality, as follows:

$$
\hat{\theta}=\tau-\frac{\sigma}{A}(\hat{p}(\theta)-B) .
$$

An efficient estimator for $p(\theta)$ can be found using a number of techniques. Here, we consider the estimator derived using the concept of sufficient statistics. By the Neyman-Fisher Factorization theorem [5], the sufficient statistic, $T(x)$, for this pdf can be determined as follows:

$$
\begin{aligned}
p(\mathbf{b} ; p(\theta)) & =\prod_{n=0}^{N-1} p(\theta)^{b(n)}(1-p(\theta))^{1-b(n)} \\
& =p(\theta)^{\sum_{n=0}^{N-1} b(n)}(1-p(\theta))^{\sum_{n=0}^{N-1}(1-b(n))} \\
& =p(\theta)^{\sum_{n=0}^{N-1} b(n)}(1-p(\theta))^{N-\sum_{n=0}^{N-1} b(n)} \\
T(x) & =\sum_{n=0}^{N-1} b(n) .
\end{aligned}
$$

This widely known result is a complete sufficient statistic. With this knowledge, the Rao-Blackwell-Lehmann-Scheffe theorem [5], states that a valid unbiased estimator of $p(\theta)$ can be found by determining which function, $g(T(X))$, results in an unbiased estimate of $p(\theta)$. Since we have

$$
\begin{aligned}
& E[T(x)]=N p(\theta) \\
& g(T(x))=\frac{1}{N} T(x),
\end{aligned}
$$

we obtain the unbiased estimate of $p(\theta)$ as

$$
\hat{p}(\theta)=\frac{1}{N} \sum_{n=0}^{N-1} b(n)=\bar{b} .
$$

Substituting (10) into (9), we arrive at the new estimator

$$
\hat{\theta}=\tau-\frac{\sigma}{A}(\bar{b}-B)
$$

where $\sigma$ is the standard deviation of the noise, $\tau$ is the common threshold value, and $A$ and $B$ are as previously defined.

\section{Performance of the New Estimator}

The first step in evaluating the performance of this new estimator is determining its bias as

$$
\begin{aligned}
E[\hat{\theta}] & =\tau-\frac{\sigma}{A}(E[\bar{b}]-B) \\
& =\tau-\frac{\sigma}{A}(p(\theta)-B) .
\end{aligned}
$$

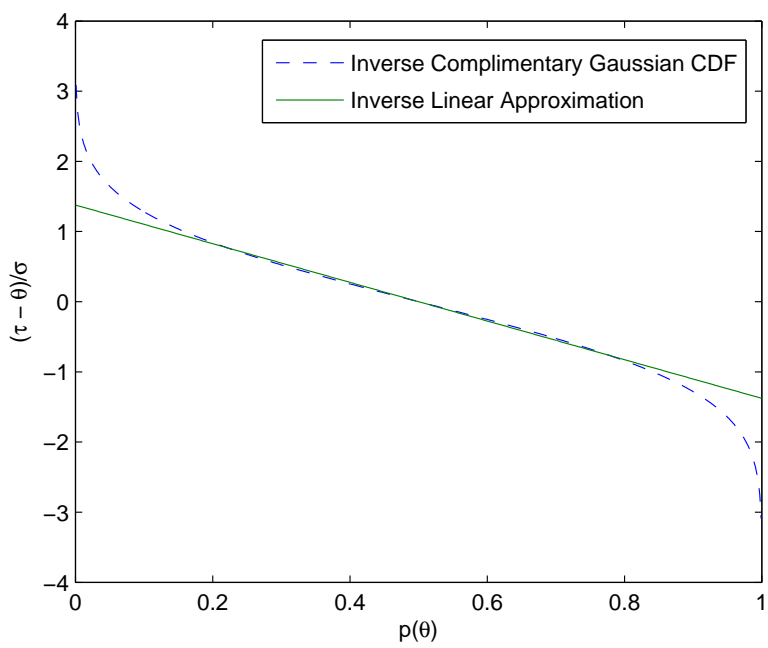

Fig. 2. The complimentary Gaussian CDF inverse and the linear approximation inverse. When $\bar{b} \leq .2$ or $\bar{b} \geq 0.8$, the proposed estimator produces an estimate with far less variance. For $\left|\frac{\tau-\theta}{\sigma}\right| \in(0.5,1), \bar{b}$ will fall into these ranges with increasing frequency.

In general, (12) is not equal to $\theta$, so the proposed estimator is biased. As such, the CRLB as derived in (6) does not bound the performance of our estimator. The appropriate performance metric to consider for this case is the mean-squared error (MSE), which can be found as follows:

$$
\begin{gathered}
E\left[(\theta-\hat{\theta})^{2}\right]=E\left[\theta^{2}\right]-2 E[\theta \hat{\theta}]+E\left[\hat{\theta}^{2}\right] \\
E\left[\hat{\theta}^{2}\right]=\operatorname{var}(\hat{\theta})+E[\hat{\theta}]^{2} \\
E\left[(\theta-\hat{\theta})^{2}\right]=\theta(\theta-2 E[\hat{\theta}])+\frac{\sigma^{2}}{A}\left(\frac{p(\theta)(1-p(\theta))}{N}\right)+E[\hat{\theta}]^{2}
\end{gathered}
$$

where $p(\theta)$ is defined in (2). The performance of this proposed estimator can then be easily compared with the performance of the MLE proposed in [4] by plotting MSE/CRLB for particular $\mathrm{N}$ values. Fig. 3 plots the MSE of the new estimator, the Chernoff (upper) bound on the performance of the MLE, the CRLB (lower) bound on the performance of the MLE, and the CRLB of the clairvoyant estimator for $N=50$. We can see that the new estimator vastly outperforms the MLE when $\left|\frac{\tau-\theta}{\sigma}\right| \in(0.45,1)$, while only slightly sacrificing performance when $\left|\frac{\tau-\theta}{\sigma}\right| \in(0,0.45)$. The reason for this is clear when we consider the inverse complimentary Gaussian CDF curve compared with the inverse linear approximation curve, which are both plotted in Fig. 2. As $(\tau-\theta) \rightarrow \sigma / 2, \bar{b}$ falls in the range $(0, .2)$ or $(.8,1)$ with increasing frequency. In these regions, the inverse Gaussian CDF curve approaches $\pm \infty$, which results in large estimation errors. Because this estimator is only appropriate for use in cases when the dynamic range of $\theta$ is small compared with $\sigma$, the very large (small) estimates of $\tau-\theta$ which the inverse complimentary Gaussian CDF produces when $|\tau-\theta| \in(.5,1)$ are inappropriate. The proposed estimator mitigates the effect of these outlier $\bar{b}$ values, resulting in better overall performance through the desired estimation range $|\tau-\theta| \leq \sigma$. 


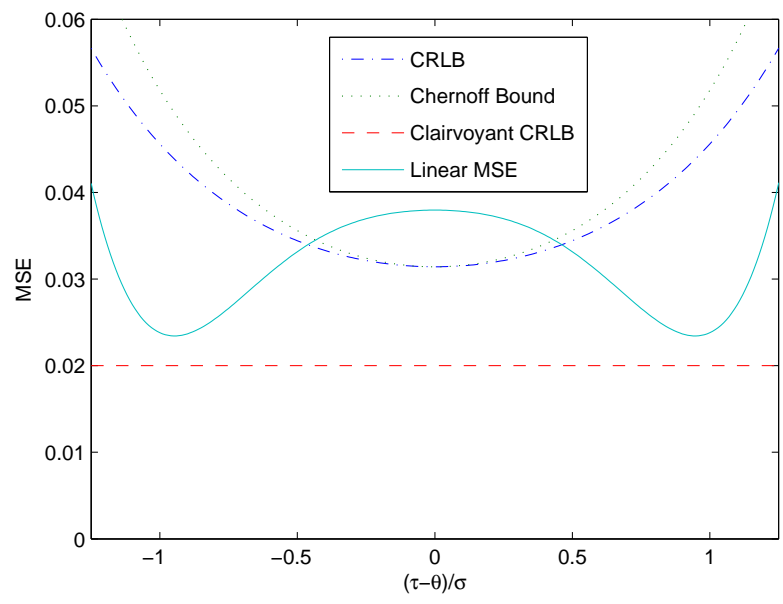

Fig. 3. The CRLB and Chernoff bound for the MLE, which serve as lower and upper bounds, respectively, on the MLE performance, as well as the CRLB of the clairvoyant estimator $\frac{1}{N} \sum_{n=0}^{N-1} x(n)$, compared with the analytical MSE of the proposed linear approximated estimator, evaluated for $N=50$.

\section{Simulation Results}

The performance of the proposed estimator based on the linear approximation to the complimentary Gaussian CDF has been analytically predicted by the MSE evaluation. However, it is imperative to validate these results through simulation. This will be accomplished by three setups: simulation for various $\frac{\tau-\theta}{\sigma}$ values with a fixed $N$, simulation for various $N$ with fixed $\frac{\tau-\theta}{\sigma}$, and simulations for randomly distributed $\tau-\theta$. The first setup permits evaluation of the correctness of the MSE derived in (13), the second demonstrates the performance of the system for a particular realization of $(\tau-\theta) / \sigma$ with differing numbers of sensors in the network, and the third allows us to consider practical realizations of the system by treating $\theta$ as random.

1) Validating the MSE Curve: For validation, we set $N=$ $50, \tau-\theta \in(-2,2)$, and $\theta=0$. Each $\frac{\tau-\theta}{\sigma}$ value is evaluated with $10^{4}$ simulation trials, with the mean of these trials recorded. Fig. 4 presents the results of this simulation. The empirical results fit the analytical curve so precisely that the subtle differences between the two are difficult to see.

2) System Realizations: Values of $\theta=1, \sigma=1, \tau=$ $\theta+\sigma[0,0.5,0.7]$, and $N=[51,52, \ldots, 100]$ are used in these simulations. Smaller values of $N$ are not used because outliers, $\bar{b}=0$ or $\bar{b}=1$, which occur with greater frequency as $N \rightarrow 0$, result in unrealizable (infinite-valued) estimates of $\theta$ for the MLE, which is also simulated to compare with the proposed estimator. For each value of $N$, the simulation is run $10^{4}$ times and the mean result is plotted. Figs. 5, 6, and 7 present these simulation results for $\frac{\tau-\theta}{\sigma}=0,0.5$, and 0.7 , respectively. For $\frac{\tau-\theta}{\sigma}=0$, the proposed estimator sacrifices estimation performance at the advantage of much lower complexity. For $\frac{\tau-\theta}{\sigma}=0.5$ and 0.7 , the proposed estimator achieves better MSE performance than the MLE while maintaining the complexity advantage. The plots show close agreement with the analytical performance illustrated in Fig. 3.

3) Overall Performance: If we assume $(\tau-\theta)$ is uniformly distributed in the range $(-\sigma, \sigma)$, the average performance

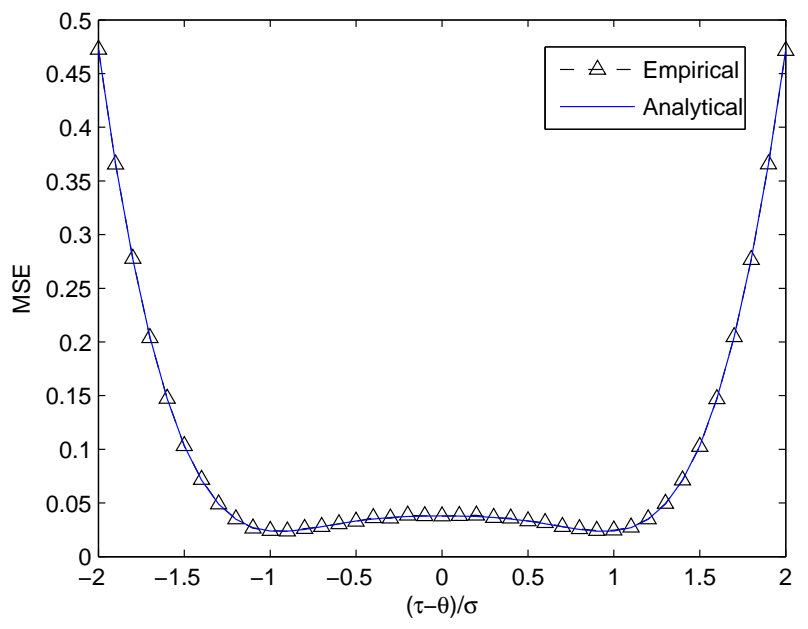

Fig. 4. Empirical and analytical MSE of the proposed estimator. The curves fit so closely that differences are nearly indiscernible.

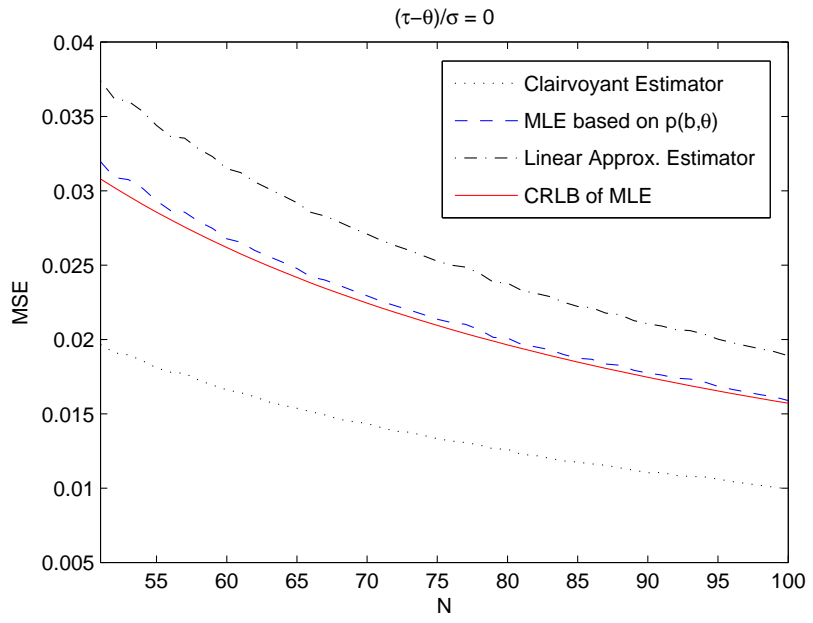

Fig. 5. The comparison, for $\frac{\tau-\theta}{\sigma}=0$, of the MSE for the MLE, clairvoyant, and proposed linear approximated estimators, as well as the CRLB of the MLE estimator.

of the MLE and proposed estimator can be computed by averaging each curve, as plotted in Fig. 3, over $(\tau-\theta)$ for the considered range. The average performance is plotted in Fig. 8. Likewise, we can consider $(\tau-\theta)$ to be Gaussian-distributed with standard deviation $\sigma_{s}=\frac{1}{3}$ and compute an average performance of each estimator. The average performance for the Gaussian distributed case is plotted in Fig. 9. The estimator based on our linear approximation to the complimentary Gaussian CDF performs better than the MLE proposed in [4], under the condition that $(\tau-\theta)$ is uniformly distributed in the range $(-\sigma, \sigma)$. When $(\tau-\theta)$ is Gaussian-distributed in the same range, the estimator based on our approximation performs nearly as well as the MLE.

\section{CONCLUSiON}

The MLE estimator proposed in [4] provides an analytically elegant solution for the bandwidth-constrained distributed estimation problem, but is computationally expensive to im- 


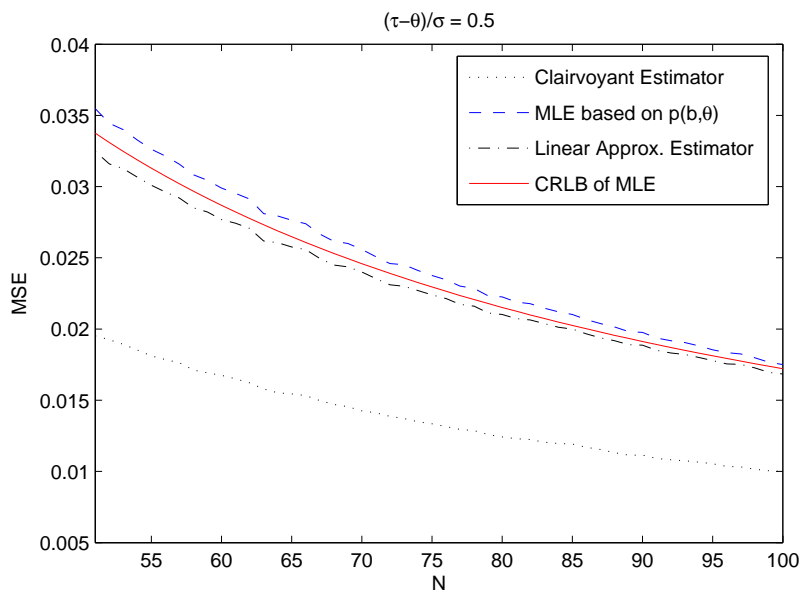

Fig. 6. The comparison, for $\frac{\tau-\theta}{\sigma}=0.5$, of the MSE for the MLE, clairvoyant, and proposed linear approximated estimators, as well as the CRLB of the MLE estimator.

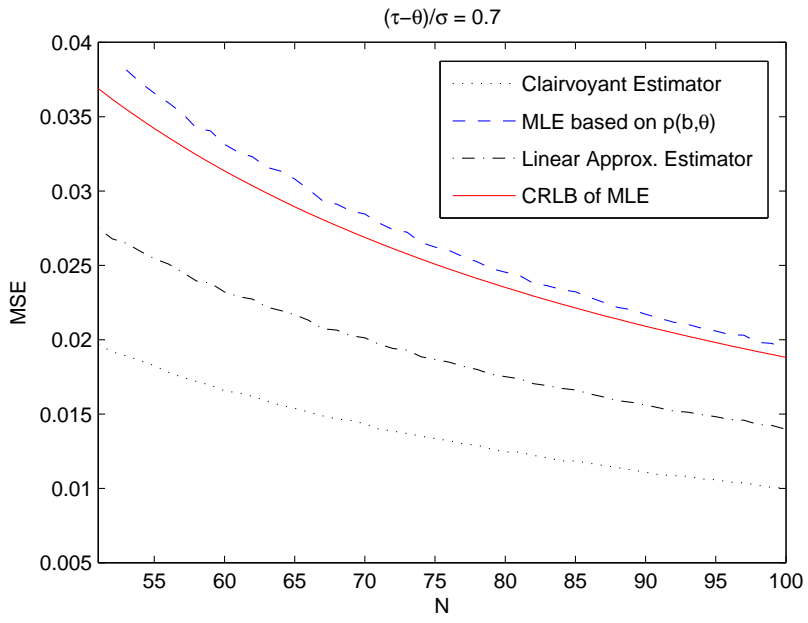

Fig. 7. The comparison, for $\frac{\tau-\theta}{\sigma}=0.7$, of the MSE for the MLE, clairvoyant, and proposed linear approximated estimators, as well as the CRLB of the MLE estimator.

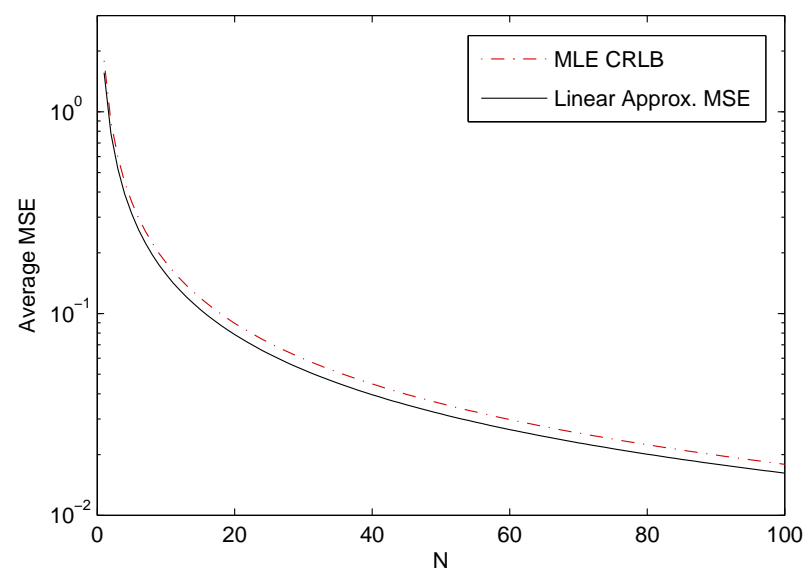

Fig. 8. CRLB of MLE and the MSE of the proposed linear approximated estimator for a random $(\tau-\theta)$ uniformly distributed in $(-\sigma, \sigma)$. The estimator based on our approximation outperforms the MLE for all $\mathrm{N}$ under this condition.

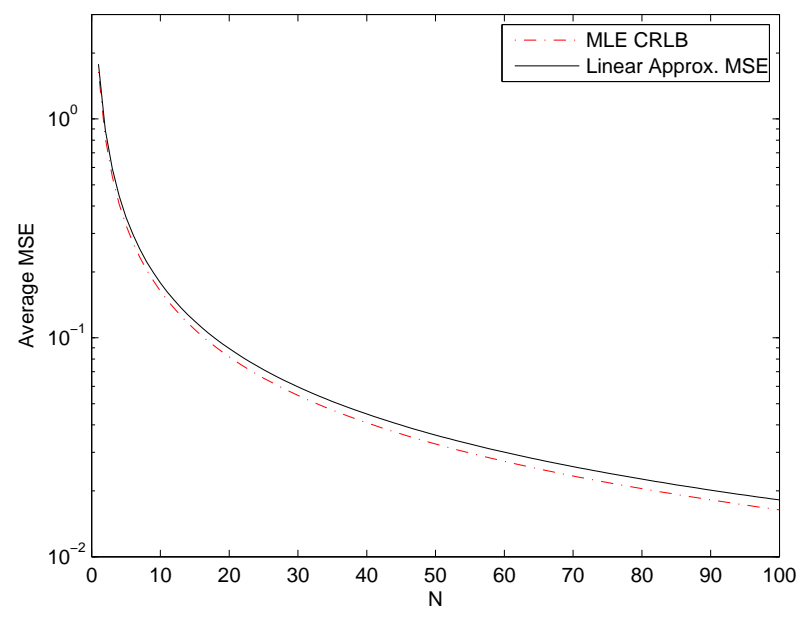

Fig. 9. CRLB of the MLE and the MSE of the proposed linear approximated estimator, for a random $(\tau-\theta)$ Gaussian-distributed with zero mean and standard deviation $\frac{1}{3}$, averaged over the considered range. The estimator based on our approximation performs nearly as well as the MLE under this condition.

plement due to its reliance on the inverse complimentary Gaussian CDF. By working within the framework established by Giannakis and Ribeiro [4], specifically that this estimator only be used when the dynamic range of $\theta$ is less than $\sigma$, a more computationally-efficient estimator may be derived which performs close to the MLE. This estimator is biased and has an MSE which is close to the variance of the MLE when $|\tau-\theta| \leq 0.45 \sigma$, and which is much lower than the variance of the MLE when $0.45 \sigma \leq|\tau-\theta| \leq \sigma$. The proposed estimator performs similarly to the MLE while providing complexity savings by eliminating dependence upon the inverse complimentary Gaussian CDF.

\section{REFERENCES}

[1] J. A. Gubner, "Distributed estimation and quantization," IEEE Transactions on Information Theory, vol. 39, no. 4, 1993.

[2] S. Kumar, F. Zhao, and D. Shepherd, "Collaborative signal and information processing in microsensor networks," IEEE Signal Processing Magazine, March 2002.

[3] Sensor Information Technology. DARPA/ITO, August 1999. [Online]. Available: http://www.darpa.mil/DARPATech2000/presentation.html

[4] A. Ribeiro and G. B. Giannakis, "Bandwidth-constrained distributed estimation for wireless sensor networks-part i: Gaussian case," IEEE Transactions on Signal Processing, vol. 52, no. 3, 2006.

[5] S. M. Kay, Fundamentals of Statistical Signal Processing. New Jersey, USA: Prentice Hall PTR, 1993. 